\title{
Transport of Bunched Beams with Space Charge through a Periodic Lattice
}

\author{
M.F.Reusch and D.L.Bruhwiler, Northrop Grumman Advanced Technology \\ and Development Center, 4 Independence Way, Princeton, NJ 08540 USA
}

We present a numerical study of beam transport through a FODO HEBT typical of proposed large current ion linear accelerator systems. Previous studies of this problem have usually assumed uniform and linear focusing forces. In contrast, our study includes non uniform focusing as well as nonlinearities associated with space charge forces, fringe fields and RF gaps. We examine current limits, beam mismatch and emittance growth. These simulations are conducted with the high order Northrop Grumman Topkark code, which implements the Garnett and Wangler 3-D space charge model. We compare our results to the analytic studies of Hofmann[3], Reiser[4] and others.

\section{INTRODUCTION}

We have used the Northrop Grumman Topkark code [1] in a preliminary study of the transport of high current intensity beams through a periodic high-energy beam transport (HEBT) lattice. Such beam transport lattices, as opposed to accelerating Linacs, form part of proposed (AXY) systems for the study of fusion materials, the accelerator production of Tritium (APT) and the accelerator transmutation of radioactive waste (ATW). Some previous studies of this problem have assumed uniform and/or linear focusing forces. In contrast, our simulation includes non uniform focusing as well as non linearities associated with space charge forces, fringe fields and RF gaps. Our principle aims are to benchmark the Topkark code in these well studied circumstances and to examine the effect that non uniform, non linear, fully $3 \mathrm{D}$ focusing and 3D, bunched-beam space charge effects have on high energy beam transport.

\section{TOPKARK}

The Topkark code used in this study is a general optics and particle tracking version implementing essentially exact dynamical applied field models along with restricted models of space charge effects. The Garnett and Wangler[2] (G\&W) space charge model used, implements a general distribution of ellipsoidal symmetry based on particle positions. Conducting wall boundary conditions are not presently implemented. The code contains combined function, i.e., including quadrupole, sextapole, octupole, etc. field moments, dipole bend and straight magnetic elements, hard and soft edge fringe field models and general misalignments[6]. The code uses both Symplectic[7] and Runge-Kutta type integrators. Topkark has been previously compared with Trace 3D in the linear limit and with Parmila, obtaining reasonable agreement. Most recently, in a paper by D. Bruhwiler and Y. Batygin at this conference, Topkark is compared to a PIC code.

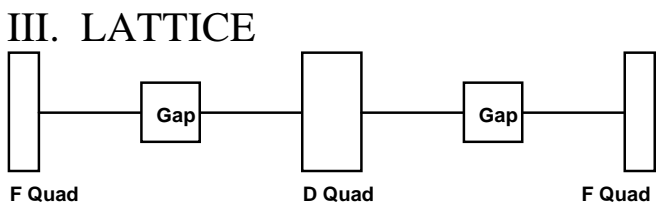

Fig. 1 - Simple FODO Lattice

For this study, we concocted a simple $\sim$ one meter cell, 10 $\mathrm{MeV}$, proton FODO lattice in which the drift spaces between the $10 \mathrm{~cm}$ focus and defocus quadrupoles are of equal length, the focus and defocus quadrupoles are of equal strength and an RF cavity is inserted between every quadrupole pair. This lattice treats the $\mathrm{X}$ and $\mathrm{Y}$ planes on an identical footing. The zero current transverse phase advance is normally arranged to be 72 degrees. The phase advances versus matched beam current with a linear space charge model are given in Fig. 2.

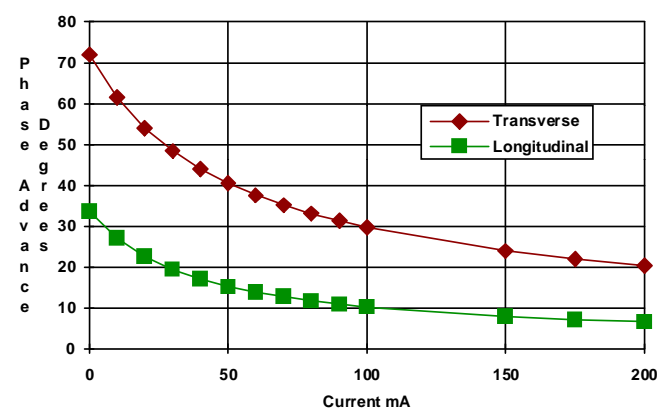

Fig. 2 - Phase advance vs current for a linear space charge model.

We confirm a number of expected phenomena in a FODO lattice with the code. The linear space charge model shows close agreement with Trace3D and exhibits very little emittance growth. With non linear space charge models, mismatched beams grow rapidly in emittance, as do unstable beams in a lattice whose zero current phase advance exceeds 90 degrees.

No precise upper limit for current is found in quiescient transport. Instead, a practical limit is provided by aperture as the matched beam size, for fixed transverse focusing strength, grows with current.

Figures 5 through 8, show the rms local transverse emittances when a matched, $100 \mathrm{~mA}$ beam with 2000 particles is propagated through 100 periods of this lattice. This is enough current that the transverse phase advance is depressed by a factor of one half and the longitudinal by a 
factor of one third. We compare simulations with a thin, quasi-linear RF gap, Figs. 5 and 7, and a more realistic thick, single mode RF cavity, Figs. 6 and 8.

The simulation includes the non linear effects of space charge, quadrupole fringe fields and RF cavity focusing. Importantly, the emittance growth of Figs. 6 and 8 is almost entirely due to the thick RF cavity and, in magnitude, is in rough agreement with an analytical estimate [8].

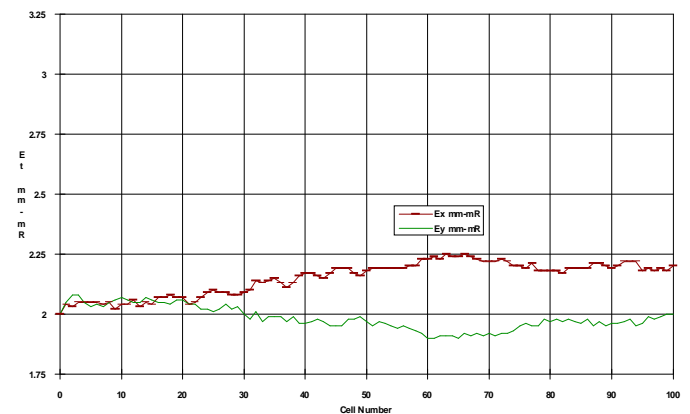

Fig. 5 Transverse Emittance vs Cell No, FODO with thin RF gap.

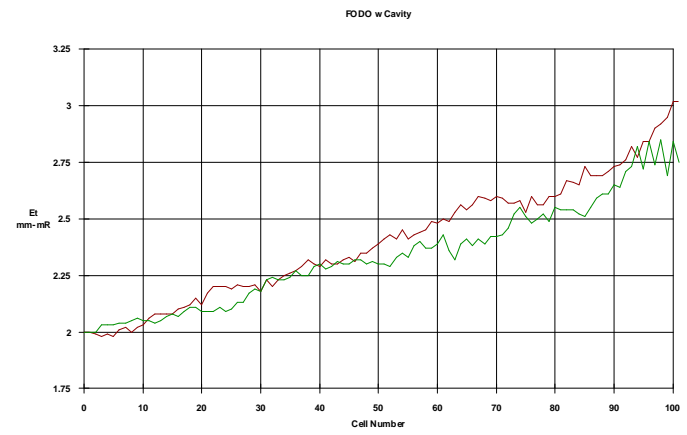

Fig. 6 - Transverse Emittance vs Cell No, FODO with thick RF cavity.

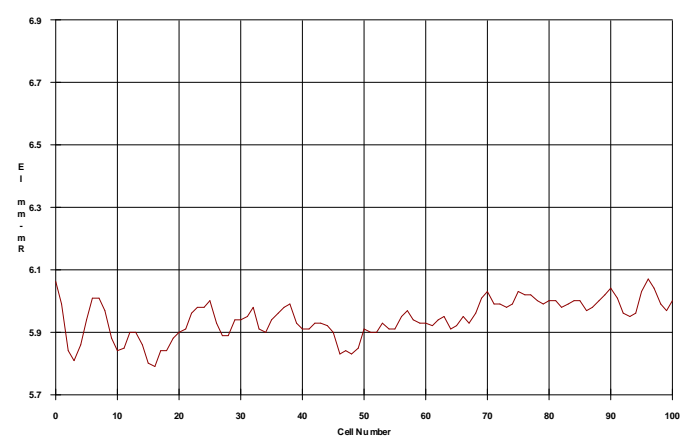

.Fig. 7 - Longitudinal Emittance vs Cell No. FODO with thin RF gap.

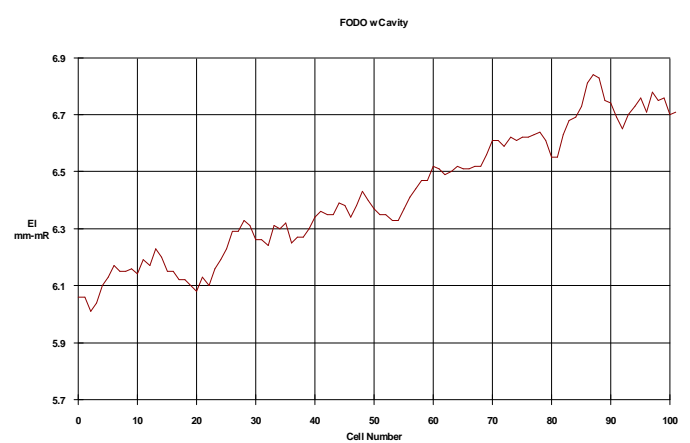

Fig. 8 - Longitudinal Emittance vs Cell No. FODO with thick RF cavity.

Multipole components in the fringe fields of the quadrupoles make very little contribution to the emittance growth suggesting that geometrical aberrations may not be significant in such HEBTs. However as more current is transported the matched beam size grows causing these non linearities to become more important.

Interestingly, in the thin gap model (Fig. 6) the transverse emittances, after tracking each other for a while, become slightly different. The reason for this transverse emittance asymmetry is not yet understood. It occurs in either plane depending on initial statistics and is not affected much by fringe fields or octupole strength in combined function magnets.

\section{BEAM MATCHING}

Ordinarily an initial upward transient of as much as $100 \%$ occurs in the emittance as a linearly matched beam is introduced into a non linear lattice. This initial transient may be regarded as spurious or not according to supposed initial conditions. However, this transient, if allowed, obscures sensitive dependencies.

The lack of initial emittance growth in the above figures has been arranged by performing a heuristic rematching procedure. In its' simplest form, the beam is propagated repeatedly through a lattice period. The emerging beam is phase-space culled of particles beyond 4 sigma. New particles are randomly introduced. The phase space is multiplicatively adjusted to preserve emittance. This preparatory "non linear matching" minimizes initial emittance growth. It allows the beam to adjust to a more or less self-consistent state.

With the G\&W model, this matching method yields beams with spatial distributions that are neither Gaussian nor uniform, but something in between, as shown in Fig. 9 . As the current is doubled from $100 \mathrm{~mA}$ to $200 \mathrm{~mA}$, for constant emittance, there is little change in the beam distribution. 


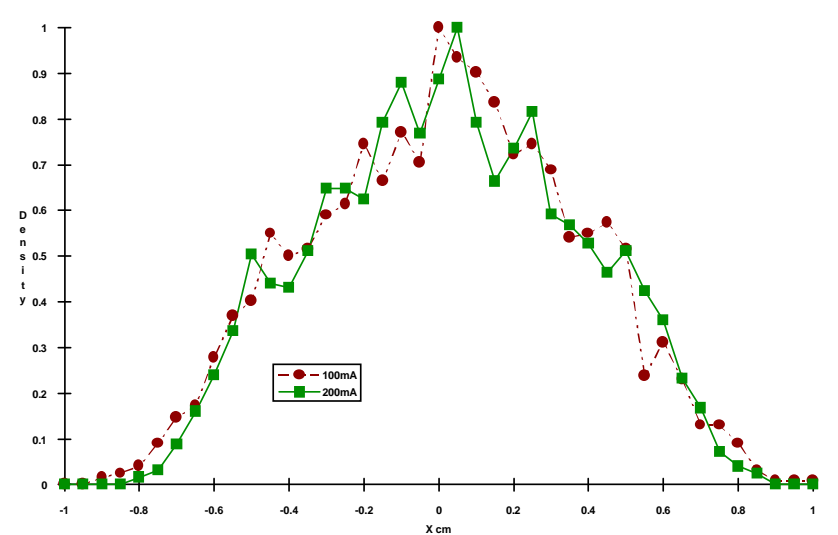

Fig. 9 - Transverse beam distributions at 100 and 200 $\mathrm{mA}$.

Figure 10 gives the "tune shift" with amplitude for an 100 $\mathrm{mA}$ beam using the G\&W model. The tunes have been deduced from an approximate transport map that has been obtained through the judicious placement of test particles and a finite difference procedure. The highly non linear G\&W space charge model emphasizes replusive space charge forces near the bunch center over the equivalent linear model. In consequence, particles near the bunch center may see an unstable lattice.

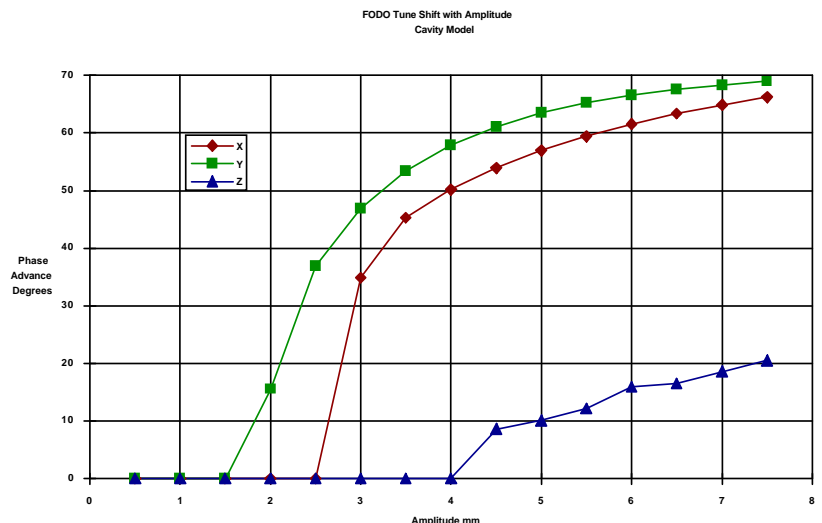

Fig. 10 - Tune shift with amplitude, G\&W space charge model at $100 \mathrm{~mA}$.

\section{CONCLUSIONS}

A preliminary study of HEBT transport and emittance growth has been carried out with the tracking version of the Northrop Grumman Topkark code. Results tend to agree with previous work encouraging the belief that Topkark will prove useful in future studies of transport lines. A non linear, emittance preserving, matching method was discussed that eliminates initial transients in bulk beam properties. With the G\&W model, this matching method yields beams with spatial distributions that are neither Gaussian nor uniform, but something in between,

\section{REFERENCES}

[1] D. L. Bruhwiler and M. F. Reusch, AIP Conf. Proc. 297, R. Ryne Editor, (AIP, New York, 1993), pp. 524531.

[2] R. Garnett and T. Wangler, IEEE Particle Accelerator Conf. Proc., (IEEE, New York, 1991), p. 330.

[3] I. Hofmann, in Applied Charged Particle Optics, Part C: Very High-Density Beams, A. Septier ed. (Academic Press, 1983).

[4] M. Reiser, Theory and Design of Charged Particle Beams (John Wiley and Sons, 1994)

[5] R. A. Jameson, Beam Intensity Limitations in Linear Accelerators, IEEE Trans. Nuclear Science, Vol. Ns-28, No. 3, June 1981, pp.2408-2412.

[6] E. Forest, M. Reusch, D. Bruhwiler and A. Amiry, Particle Accelerators, 1994, Vol. 45, pp.65-94

[7] E. Forest, J. Bengtsson, M. Reusch, Physics Letters A, 158 (1991) 99-101.

[8] M. Weiss, CERN/MPS/LI Report 73-2 Geneva (1978). 